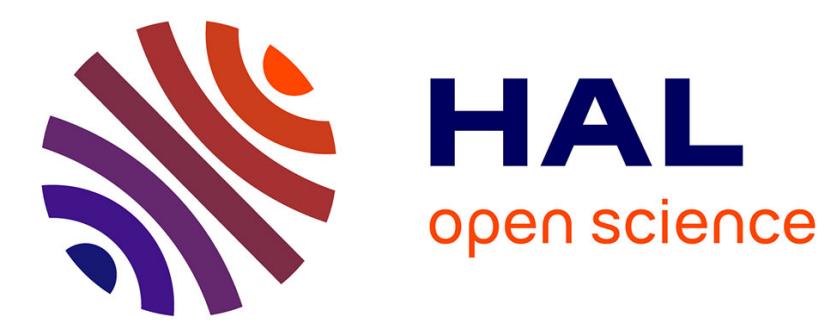

\title{
Prendre la mesure du droit: enjeux de l'observation statistique pour la sociologie juridique
}

\author{
Romain Melot, Jérôme Pélisse
}

\section{To cite this version:}

Romain Melot, Jérôme Pélisse. Prendre la mesure du droit: enjeux de l'observation statistique pour la sociologie juridique. Droit et Société, 2008, 69-70, pp.331-346. hal-01198161

\section{HAL Id: hal-01198161 \\ https://hal.science/hal-01198161}

Submitted on 29 May 2020

HAL is a multi-disciplinary open access archive for the deposit and dissemination of scientific research documents, whether they are published or not. The documents may come from teaching and research institutions in France or abroad, or from public or private research centers.
L'archive ouverte pluridisciplinaire HAL, est destinée au dépôt et à la diffusion de documents scientifiques de niveau recherche, publiés ou non, émanant des établissements d'enseignement et de recherche français ou étrangers, des laboratoires publics ou privés. 


\section{Prendre la mesure du droit : enjeux de l'observation statistique pour la sociologie juridique}

\section{Romain Melot *, Jérôme Pélisse **}

\section{Résumé}

Les données quantitatives et les outils statistiques font partie des matériaux et des techniques d'observation mobilisables par la sociologie pour comprendre les usages du droit lorsqu'ils sont envisagés comme des régularités sociales. Mais à l'exception des débats relatifs aux méthodes quantitatives appliquées à la sociologie de la déviance et de la criminalité, ces données et ces outils sont en général peu discutés dans le champ de la sociologie du droit. À partir d'exemples appliqués entre autres au droit du travail, nous proposons ici de réfléchir aux usages de ces outils statistiques en analysant le recours aux données quantitatives au croisement de la sociologie du droit et d'autres domaines de la sociologie, ainsi que les techniques d'enquêtes utilisées par les recherches empiriques.

Contentieux - Données quantitatives - Droit du travail - Méthodes statistiques.

\section{Summary}

Gauging the Law : Issues of Statistical Observation for Legal Sociology Sociologists can employ statistical tools to understand the uses of law. While often debated when applied to the sociology of deviance or criminality, these tools are rarely discussed in the sociology of law. Drawing from examples in labor law, we analyze both the recourse to quantitative data at the intersection of legal sociology and other sociological approaches, as well as the methods of inquiry employed in empirical research.

Labor Law - Litigation - Quantitative Data - Statistical Methods.

\section{Les auteurs}

\section{Romain Melot}

Chargé de recherche en sociologie à l'INRA (UMR Sadapt). Il a consacré sa thèse à l'analyse des pratiques contractuelles en matière de règlement des litiges dans le domaine du droit du travail. Ses recherches actuelles portent notamment sur l'analyse statistique du contentieux en matière civile et administrative.

\section{Jérôme Pélisse}

Maître de conférences en sociologie à l'Université de Reims, chercheur à l'IDHE (UMR 8533, ENS Cachan). Après une thèse sur les politiques de réduction du temps de travail, il a étudié les conflits du travail à partir de l'enquête REPONSE, et mène une recherche quantitative sur les experts judiciaires.

\footnotetext{
* AgroParisTech, 16 rue Claude Bernard, F-75231 Paris cedex 05.

$<$ romain.melot@agroparistech.fr>

** Institutions et Dynamiques Historiques de l'Économie (IDHE), Bâtiment Laplace, École Normale Supérieure, 61 avenue du Président Wilson, F-94235 Cachan cedex.

$<$ jpelisse@idhe.ens-cachan.fr>
} 
Si la question méthodologique de la mesure de la délinquance et de la criminalité alimente régulièrement les controverses entre spécialistes de sciences sociales, il est moins fréquent que soit abordée, du moins en France, la problématique plus générale de l'évaluation statistique du droit, notamment lorsqu'elle s'intéresse à des domaines autres que celui du droit pénal. Il ne semble donc pas superflu de proposer, à défaut d'un bilan systématique, une réflexion " en friche » sur la production des sciences sociales en la matière, à partir d'une sélection de thématiques de recherches en sociologie incluant une analyse quantitative du droit. De manière restrictive, notre propos se limitera au champ de la sociologie du droit (en écartant, par exemple, le champ considérable de l'économie et de l'histoire du droit) et ne traitera pas des rapports entre droit et statistique dans une perspective historique. Enfin, un accent particulier sera mis sur les travaux relatifs aux usages du droit du travail, sur lequel les deux auteurs de ce texte ont développé des recherches, qu'il s'agisse des questions d'appropriation du droit dans un contexte de négociation collective ou des pratiques contractuelles 1 .

\section{Des contextes d'étude diversifiés}

Sans s'appesantir sur les liens historiques et logiques qui unissent mise en forme statistique et mise en forme légale ${ }^{2}$, on s'intéressera ici de deux manières aux contextes dans lesquels sont produites les études quantitatives s'appuyant ou traitant du droit et de la justice. La première concerne la construction des données mobilisées et la seconde les objets étudiés.

\section{l.1. Registres, enquêtes et « activités juridiques »}

La distinction analysée par Alain Desrosières entre enquête directe et registre administratif ${ }^{3}$ peut en effet trouver en France une application aux statistiques judiciaires. À côté d'une activité de production statistique rendant compte de manière périodique des flux d'affaires enregistrés par les juridictions, coexistent des enquêtes directes réalisées sur des corpus de décisions spécifiques pour des recherches ciblées ${ }^{4}$. Cette dualité des sources statistiques - registres $v s$ enquêtes - peut être analysée au regard des techniques employées (par sondage dans le second cas), mais aussi au re-

1. Jérôme PÉLISSE, « Consciences du temps et consciences du droit chez des salariés à 35 heures », Droit et Société, 53, 2003 ; Romain MELOT, «Les transactions lors de ruptures du contrat de travail », Travail et Emploi, 104, 2005.

2. Alain DeSRosIÈRES, La politique des grands nombres. Histoire de la raison statistique, Paris, La Découverte, 1993.

3. Alain DESROSIÈRES, «Enquêtes versus registres administratifs : réflexions sur la dualité des sources statistiques », Courrier des statistiques, 111, 2004.

4. Dans le champ du droit du travail, voir Évelyne SERVERIN, «Les litiges du travail au temps du jugement prud'homal », Revue nationale des barreaux, 66/67, 2002, p. 65-167; ou dans celui du contentieux du bail : Pierre ANCEL (dir.), Les décisions d'expulsion des occupants sans droit ni titre : connaissance empirique d'un contentieux hétérogène, Rapport pour le ministère de la Justice, juin 2003.

Droit et Société 69-70/2008-332 
gard de la démarche qui a présidé à la production de ces données et surtout à leurs usages : routinisé, institutionnalisé et inscrit dans l'activité administrative ou produit et utilisé pour la recherche.

L'un des principaux avantages des registres, données institutionnelles et autres enquêtes périodiques est, par exemple, de permettre des études longitudinales, ce que n'autorisent pas toujours les données issues d'une enquête ponctuelle. Les questionnements portant sur des processus et des évolutions historiques pourraient être testés de cette manière. Cependant, ces mêmes sources administratives se heurtent à un problème classique et récurrent depuis les critiques ethnométhodologiques ${ }^{5}$, souligné par Desrosières : "Ces registres reflètent-ils la société ou l'activité des administrations ? » Ce dernier propose de sortir de cet apparent paradoxe en réfléchissant aux usages des statistiques, de manière à reconstituer les relations mutuelles entre manières de penser la société, actions sur celle-ci, et modes de description. Il en déduit que « les sources administratives reflètent plutôt l'action déjà existante, tandis que les enquêtes sont plus à même d'exprimer les aspects nouveaux de la société » 6 .

Cette distinction semble se retrouver dans le domaine des statistiques judiciaires, mais aussi plus largement dans l'étude quantitative des phénomènes et des « activités juridiques » (legal activity), pour reprendre le terme utilisé par Lawrence Friedman pour définir l'ensemble des objets, dispositifs et pratiques étudiés par l'analyse empirique du droit comme phénomène social 7 . En s'inspirant de cette approche, on pourrait ainsi distinguer grossièrement quatre grandes catégories d'activité juridique.

La première concerne tout ce qui relève de la production $d u$ droit, au sens de l'activité législative et réglementaire. Dans ce domaine, les travaux de sociologie législative portant sur les luttes socio-politiques et les jeux d'acteurs présidant à la production normative sont principalement monographiques. Néanmoins, on peut citer des exemples d'analyse statistique comme les recherches portant sur des corpus de création d'infractions sur une période déterminée, et se fondant sur le recensement de textes législatifs et réglementaires 8 .

Une autre catégorie englobe le champ très vaste de "l'activité décisionnelle » qui renvoie par excellence à l'activité des tribunaux, laquelle peut être étudiée de manière quantitative aussi bien par l'analyse statistique du contentieux que par le recours à différents outils d'analyse de la jurispru-

5. John Kitsuse et Aaron CiCOUREL, « Note on the Use of Official Statistics », Social Problems, 11, 1963.

6. Alain DESROSIÈRES, «Enquêtes versus registres administratifs : réflexions sur la dualité des sources statistiques », op. cit.

7. Lawrence FrIEDman, The Legal System : A Social Science Perspective, New York, Russel Sage Foundation, 1975.

8. Cécile BARBERger et Pierre LASCOUMES, Le temps perdu à la recherche du droit pénal. Analyse de cinq années de textes comportant des dispositions pénales 1983-1987, Paris, GAPP-CNRSMinistère de la Justice, 1991.
Prendre la mesure du droit : enjeux de l'observation statistique pour la sociologie juridique 
dence (statistique textuelle sur les registres d'argumentation, par exemple ${ }^{9}$ ). En outre, ce type d'activité décisionnelle est la production principale de nombreuses institutions qui, du fait de leurs compétences, sont amenées à traiter régulièrement de séries de demandes auxquelles elles répondent par des décisions d'allocation de droits. Il peut s'agir de " quasi-juridictions », ayant des similitudes de fonctionnement avec les tribunaux mais sans en suivre les procédures 10, d'institutions administratives (services déconcentrés de l'État ou des collectivités locales, commissions diverses intégrant plus ou moins des acteurs non administratifs - associatifs ou professionnels) qui se prononcent sous la forme d'avis ou de décisions dans différentes matières (contrôle de légalité, autorisations diverses, injonctions). D'autres enfin relèvent de la sphère privée, mais de par leur taille critique et leur caractère bureaucratique se rapprochent de ce modèle d'activité décisionnelle routinisée (les services de ressources humaines ou les services clientèles et commerciaux de grandes entreprises qui traitent des flux quotidiens de litiges, par exemple).

Une troisième catégorie renvoie aux pratiques liées à l'usage d'outils juridiques: les usages et la production de contrats divers (contrats commerciaux, contrats de travail) ou d'autres actes juridiques (comme les actes notariés), des règlements privés (règlements intérieurs d'associations ou d'entreprises), ou des pratiques relatives à la négociation collective ${ }^{11}$ sont autant d'objets possibles pour des investigations statistiques. Lorsqu'il s'agit d'actes privés, ils peuvent cependant poser des difficultés d'accès, liées au caractère confidentiel ou à l'absence d'enregistrement obligatoire de la plupart de ces documents, et nécessitent la mise au point de méthodes de collectes originales pour constituer un corpus susceptible d'être soumis à un traitement quantitatif 12 .

Enfin, on peut définir les contours d'une dernière catégorie, plus mouvante et potentiellement ouverte, qui intégrerait l'ensemble des représentations du droit et des comportements et actions "orientés vers le droit ", dont l'objectivation passe souvent par des techniques d'observation et d'entretien - comme dans la majorité des legal consciousness studies 13 - mais aussi, parfois, par l'analyse de questionnaires.

\footnotetext{
9. Claude DIDRY, «Les comités d'entreprise face aux licenciements collectifs : trois registres d'argumentation », Revue française de sociologie, 29 (3), 1998.

10. Cf. sur ce point les enquêtes sur les institutions de traitement des réclamations mises en place dans les organismes de sécurité sociale : Isabelle SAYN, «Accès au juge et accès au droit dans le contentieux de la protection sociale », Revue française des Affaires sociales, 3, 2004.

11. Voir Olivier BARRAT et Catherine DANIEL, « La négociation collective, le statisticien, sa lanterne et le débat social », Revue de l'IRES, 39, 2002.

12. Voir Christian BESSY, La contractualisation de la relation de travail, Paris, LGDJ, coll. « Droit et Société », 2007, qui construit depuis plusieurs années une base originale de contrats de travail. Voir aussi Romain MELOT, «Les transactions lors de ruptures du contrat de travail », op. cit.

13. Voir Jérôme PÉLISSE, «A-t-on conscience du droit? Autour des "legal consciousness studies" », Genèses, 59, 2005.
} 


\section{I.2. La question du cadrage juridique des objets}

Une autre distinction concerne moins les données, leur production et leurs usages, que les questions que se posent les chercheurs, en prenant en compte le fait que ces données sont justement «cadrées juridiquement » ou non au départ de la recherche.

Les uns peuvent en effet recourir à des sources juridiques pour y puiser des informations sociales, économiques, ou culturelles. L'analyse d'un corpus d'actes testamentaires a ainsi ouvert celle des représentations de la mort de 1300 à nos jours, sans que l'étude prenne spécifiquement en compte l'usage juridique des clauses de ces actes ${ }^{14}$. Les archives judiciaires ont pu être utilisées pour analyser les mentalités et réalités économiques ou sociales passées. Les classes populaires ont été bien souvent étudiées uniquement par ce prisme judiciaire et même pénal - car ces sources, pour diverses raisons, sont les seules qui en "parlent »-, ce qui n'a pas été sans susciter des réflexions quant à la vision que les historiens pouvaient avoir de ces classes populaires 15 . Ainsi, de nombreuses recherches ont utilisé des données juridiques et/ou judiciaires pour s'intéresser à tout autre chose que le droit ou la justice, tant le droit peut être considéré, selon la célèbre formule de Durkheim dans De la division du travail social, "comme un symbole de la solidarité sociale». La sociologie de la famille recourt par exemple quotidiennement à des statistiques juridiques à travers des nombres de mariages, divorces, adoptions qui sont autant de faits sociaux renseignés, sinon même existant, par leurs traitements juridiques et éventuellement judiciaires. Cette conception sociologique du droit comme «social cristallisé » a ainsi ouvert un usage avant tout méthodologique des données judiciaires ou juridiques.

Cependant, utiliser ces données institutionnelles (lois, normes, règlements, décisions de justice, actes juridiques, etc.) pour parler d'autre chose - en particulier lorsqu'on les construit sous un angle quantitatif en comptabilisant des infractions, des PACS, des baux ou des accidents du travail n'est pas sans risque si l'on oublie le lien entre nomenclature statistique et mise en forme légale. En effet, comme le souligne Michel Gollac, ce lien «a parfois été interprété, à tort, comme une incitation à subordonner la méthode statistique à l'existence d'une mise en forme juridique. Or d'une part, une bonne loi suit les mœurs : elle s'appuie sur une réalité sociale durcie, donc déjà susceptible d'inscription statistique. D'autre part, toute forme légale n'est pas la bonne forme. Si le droit est un point d'appui commode pour la collecte des données, leur usage peut exiger de toutes autres

14. Michel Vovelle, Piété baroque et déchristianisation en Provence au XVIII siècle. Les attitudes devant la mort d'après les clauses des testaments, Paris, Plon, 1972.

15. Arlette FARGE, Le goût de l'archive, Paris, Seuil, 1989. Cf. également la récente thèse de Deborah COHEN, «Le peuple : de l'Autre au différent. La construction des identités individuelles et collectives des classes populaires (France, XVIII ${ }^{e}$ siècle) », qui exploite principalement des registres de demandes de grâce.
Prendre la mesure du droit : enjeux de l'observation statistique pour la sociologie juridique 
connexions. [Ainsi], une statistique peut reposer sur un socle solide, "durci" par le droit, et n'être pas pour autant utilisable d'une part ; et inversement, la statistique peut même créer de la réalité [voire] acquérir une force quasilégale, tel l'indice des prix »16. Il y a donc deux manières pour une statistique de "tenir» : s'appuyer sur une objectivation préalable des objets fixés (grâce au droit mais aussi à des conventions non juridiques - par exemple, le sexe) ; ou s'insérer dans un réseau d'usages et d'utilisateurs - quand une variable " marche bien ", c'est à dire se relie aisément à d'autres savoirs. Du coup, «l'obligation d'un appui sur le droit disparaît si on ne considère pas la statistique comme devant être une image fidèle de la réalité (quitte à forger la réalité si besoin est) mais comme un dispositif expérimental parmi d'autres (poser une question, ce n'est pas extraire un morceau de la réalité, c'est se livrer à une expérience sociale) »17. Ainsi, dans le cas des conditions de travail, se caler sur les seules catégories juridiques (par exemple, pour compter les accidents du travail ou mesurer les maladies professionnelles), c'est méconnaître les acquis de l'ergonomie ou de la sociologie du travail et manquer de pertinence scientifique.

D'une manière générale, le champ des données utilisables pour comprendre les activités juridiques ne se limite pas à celles produites par l'administration judiciaire, qu'il s'agisse de recherches s'appuyant sur des registres, des enquêtes institutionnalisées et routinisées, ou sur des enquêtes ponctuelles construites uniquement dans un objectif de recherche. L'enquête périodique du ministère du Travail sur les conditions d'emploi de la main d'œuvre permet par exemple, entre autres, de comptabiliser le nombre de contrats nouvelle embauche (CNE) signés dans les entreprises ou de mesurer l'évolution de la durée du travail collective affichée, et ainsi de disposer d'indicateurs permettant d'évaluer, même très grossièrement, la diffusion de normes législatives et leur appropriation différentielle selon les secteurs, les lieux ou le type d'acteurs. Un phénomène comme la forte augmentation de la part des licenciements pour motif personnel au détriment des licenciements pour motif économique suscite, par exemple, des réflexions croisées entre les études sur le marché du travail et celles sur le contentieux ${ }^{18}$. Des enquêtes routinisées de ce type (quasi-registres intégrés à la production habituelle de chiffres intéressant l'administration), a priori orientées par d'autres questions que celles touchant spécifiquement aux activités juridiques, comprennent ainsi des données quantitatives utiles pour évaluer des politiques publiques ou saisir des pratiques orientées par le droit.

Dans ce contexte d'étude, les représentations du droit et de la justice sont tout aussi concernées : l'enquête permanente sur les conditions de vie

16. Michel Gollac, « Donner un sens aux données. L'exemple des enquêtes statistiques sur les conditions de travail », Dossier du Centre d'Études de l'Emploi, 3, 1994.

17. Ibid.

18. Maria-Térésa Pignoni et Patrick ZouARY, « Les nouveaux usages du licenciement pour motif personnel », Premières informations et Premières synthèses, n²8.2, juillet 2003. 
de l'INSEE permet d'étudier la perception et les sentiments de sécurité et d'insécurité et les comportements de dépôts de plainte (ou non) de la population 19. De même, des enquêtes ponctuelles comme celle que Louis Pinto mène à partir de questionnaires portant sur des "pépins de consommation » 20 permettent aussi d'analyser «le sens juridique ordinaire » - cette «base de la pyramide » qui fait passer des «problèmes » aux litiges 21 - qui s'avère si difficilement saisissable, tout en posant " au droit un problème qui n'est autre que celui des conditions de son appropriation effective ${ }^{22}$. Dans une autre veine, l'enquête « RTT et Mode de vie » interrogeant en 2001 des salariés sur leurs perceptions du passage à 35 heures ouvre bien des questions de sociologie du droit lorsque les auteurs qui l'ont exploitée remarquent que " le sentiment global d'amélioration de la vie quotidienne (au travail et en dehors) est étroitement lié au respect de "l'esprit" de la loi » 23. Enfin, il va de soi que les enquêtes prosopographiques ou utilisant des questionnaires pour cerner la régularité des pratiques des professionnels ou quasi-professionnels du droit (conseillers prudhommaux 24 , avocats 25 , huissiers de justice 26 , experts judiciaires 27 , etc.) sont également des sources quantitatives précieuses pour étudier le fonctionnement de la justice et la place du droit dans la société.

\section{I.3. Élargir le spectre des objets pour l'analyse statistique : à la recherche des OJNI (objets juridiques non identifiés)}

L'analyse quantitative du droit amène par conséquent le chercheur à se poser deux questions conjointes à propos des objets qu'il étudie. La première est celle de la qualification de l'objet empirique comme objet juridique. Pour l'historien des mentalités, le testament constitue ainsi un docu-

19. Sans entrer dans le vif débat qui entoure ce type de données, voir par exemple Emmanuelle CRENNER, "Insécurité et sentiment d’insécurité », INSEE Première, 501, 1996, qui exploite dans la perspective des études de victimation des données de l'enquête INSEE.

20. Louis PINTO, «Du "pépin” au litige de consommation. Une étude du sens juridique ordinaire », Actes de la recherche en sciences sociales, 76/77, 1989.

21. Voir, sur ce point, William FELSTINER, Richard ABEL et Austin SARAT, «The Emergence and Transformation of Disputes : Naming, Blaming, Claiming », Law and Society Review, 15 (3-4), 1980-1981.

22. Louis PINTO, «Du "pépin” au litige de consommation. Une étude du sens juridique ordinaire », op. cit.

23. Marc Antoine ESTRADE, Dominique MEDA et Renaud ORAIN, « Les effets de la réduction du temps de travail sur les salariés : qu'en pensent les salariés un an après? », Premières synthèses $\mathrm{n}^{\circ}$ 21.1, 2001. Bien sûr, la recherche ne fait que commencer après cet énoncé pour un sociologue du droit.

24. Hélène Michel, «Les carrières prud'homales au prisme de la justice. Distance au rôle judiciaire et redéfinition des pratiques sociales », in Hélène Michel et Laurent WiLlEMEZ (dir.), La justice au risque des profanes, Paris, PUF/CURAPP, 2008.

25. Lucien KARPIK, Les avocats entre l'État et le marché, Paris, Gallimard, 1996.

26. Alexandre MAtTHIEU-Fritz, Les huissiers de justice, Paris, PUF, 2005.

27. Laurence Dumoulin, L'expert dans la justice, Paris, Economica, coll. «Études politiques », 2007.
Prendre la mesure du droit : enjeux de l'observation statistique pour la sociologie juridique 
ment permettant d'analyser des représentations de la mort 28 . Pour le sociologue du droit ou l'historien du droit au contraire, c'est l'étude des clauses de l'acte juridique testamentaire en usage qui seront la matière de l'analyse. Un même document peut donc être appréhendé par rapport à sa qualité d'objet juridique ou en faisant abstraction de celle-ci, suivant la perspective de recherche adoptée.

La seconde question est celle du caractère quantifiable des objets étudiés. Cette question associe étroitement la nature de l'objet à son contexte de production. L'institutionnalisation du cadre d'enregistrement est un trait des procédures juridiques, dont le caractère routinisé a très tôt intéressé les sociologues, soit parce qu'il constitue une manière de stabiliser des situations d'observations dans une perspective monographique (les premières enquêtes ethnométhodologiques de Garfinkel portent sur l'observation d'audiences judiciaires, de nombreuses recherches sociologiques et anthropologiques portent sur les interrogatoires de police), soit parce qu'il donne prise à une observation sérielle.

Un dossier administratif ou des correspondances entre un service administratif et un particulier peuvent, par exemple, faire l'objet d'une analyse purement monographique. Mais dans la mesure où ces documents font l'objet d'une production inscrite dans une procédure (l'obligation de motiver une décision administrative, par exemple) ou même simplement routinisée, ils peuvent être susceptibles d'un enregistrement ou d'un archivage systématique qui en transforme la nature : de matériaux à usage monographique, ils deviennent des séries quantifiables, comme nous l'avons évoqué plus haut à propos des documents contractuels 29. Dans un domaine touchant aux usages juridiques de l'espace (droit de l'urbanisme), des recherches ont même pu traiter de manière statistique des documents graphiques (plans de zonage), considérés comme des objets juridiques ${ }^{30}$. L'analyse sérielle d'interactions verbales dans un cadre administratif peut également faire l'objet d'une objectivation statistique dès lors que la formalisation de l'interaction le permet ${ }^{31}$. Certains OJNI - « objets juridiques non identifiés » - du point de vue des travaux de doctrine deviennent ainsi des supports d'analyse authentiques pour la compréhension des usages du droit.

\footnotetext{
28. Michel Vovelle, Piété baroque et déchristianisation en Provence au XVIII siècle. Les attitudes devant la mort d'après les clauses des testaments, op. cit.

29. Voir Romain Melot, « Les transactions lors de ruptures du contrat de travail », op. cit.

30. Paul AmY dans Espaces agricoles et urbanisme, Paris, L'Harmattan, 1996.

31. Isabelle SAYN, «L'accueil et le traitement des réclamations des allocataires par les caisses d'allocations familiales », Recherches et Prévisions (CNAF), 73, 2003.
} 


\section{La question de l'évaluation}

\section{II.1. Le contexte du débat et les controverses}

La recherche statistique portant sur l'activité juridique est donc loin d'être dépourvue de matériaux d'enquêtes. D'une certaine manière, c'est l'ensemble des objets juridiques qu'il s'agit de redécouvrir du point de vue de leur intérêt pour l'analyse quantitative. À cette richesse des matériaux s'opposent par contraste, d'une part, l'usage souvent frustre, désincarné ou souvent hors de propos qui est fait des statistiques administratives relatives à la justice dans le débat public, et d'autre part - ce qui est de loin le plus récurrent - la fréquence de jugements évaluatifs «sous-jacents » associant généralement à l'évaluation pseudo-objective une évaluation normative ${ }^{32}$.

Une des caractéristiques de ce "prêt à penser » est précisément d'occulter les usages et intérêts sociaux des dispositifs juridiques dont on «constate » l'évolution. Les jugements évaluatifs faisant apparaître et condamnant par exemple « l'explosion du contentieux », outre le fait qu'ils occultent le caractère contrasté des données existantes sur le sujet, posent bien plus la question en termes de psychologie ordinaire - comme celles portant sur le comportement supposé de plus en plus "procédurier » des individus - que de logiques sociales (lesquelles s'expriment souvent dans le système judiciaire au travers de procédures individuelles). Car l'intérêt à recourir au tribunal n'est bien sûr pas le même pour une société de crédit que pour un demandeur occasionnel. De même, la dénonciation de "l'inflation législative » fait trop souvent l'économie d'une réflexion sur les «destinataires» des textes, qui souvent ne sont autres que les «demandeurs». Pierre Lascoumes et Cécile Barberger 33 montrent ainsi, au travers de leur étude statistique de la production d'infractions, ce phénomène de circularité suivant lequel, d'une part, la plupart des règles pénales instaurées le sont à destination d'un "public » de professionnels dans un cadre technique extrêmement resserré et, d'autre part, ceux qui demandent et obtiennent la production de règles sont précisément les destinataires des dispositifs instaurés (en l'occurrence les services administratifs qui souhaitent le renforcement de leur outillage répressif).

Pour proposer un usage adéquat des données statistiques appliquées à l'activité juridique, il importe donc de replacer ces différents objets de débat dans le cadre d'une discussion scientifique. Celle-ci doit s'interroger sur le type de données et les niveaux d'observation pertinents, ainsi que sur les catégories d'usagers du droit associées à des catégories de normes mobilisées, sachant que les dispositifs juridiques définissent souvent en amont

32. Pierre Lascoumes et Évelyne Serverin, « Théories et pratiques de l'effectivité du droit », Droit et Société, 2, 1986.

33. Cécile BARBERgER et Pierre LASCOUMES, Le temps perdu à la recherche du droit pénal. Analyse de cinq années de textes comportant des dispositions pénales 1983-1987, op. cit.
Prendre la mesure du droit : enjeux de l'observation statistique pour la sociologie juridique 
les catégories d'acteurs susceptibles d'être observés a posteriori dans des situations (contentieuses ou non) de mobilisation de la règle 34 .

\section{II.2. Construction et usage de la mesure : l'exemple du dispositif d'évaluation des lois Aubry}

Pour éclairer la production et les usages de ces données visant à mesurer, ou plutôt à évaluer le droit ou l'application des lois, les politiques de réduction du temps de travail (RTT) constituent un exemple intéressant. On peut ainsi relativiser les frontières entre registres et enquêtes, montrer la forte dépendance de ces registres vis-à-vis des variations des politiques publiques, et souligner le caractère instrumental de la plupart de ces évaluations. L'évaluation des lois Aubry a été marquée par la mise en place de plusieurs dispositifs, dont deux répondent aux doux noms d'Aglaé et de Sidonie. De fait, à l'image du titre d'une série télévisée enfantine proche, c'est un véritable manège enchanté qui se donne à voir lorsqu'on étudie cette évaluation. Manège, car des sources administratives diverses ont été mobilisées à tour de rôle, quelques enquêtes venant en renfort dans un laps de temps assez court (1999-2002) sans être renouvelées. Enchanté, car la soumission de cette évaluation au politique - en particulier celle de la première loi Aubry durant l'année 1999 - a conduit à une présentation enjolivée des 35 heures qui s'est retournée de manière tout aussi spectaculaire et a été instrumentalisée politiquement à partir de 2001-2002.

Inscrite dans une première loi d'incitation votée en juin 1998, l'évaluation des accords d'entreprise anticipant la réduction légale de la durée du travail devait nourrir une seconde loi en 2000 chargée de régler définitivement le régime juridique du temps de travail. Ces accords, déposés par les entreprises auprès des Directions départementales du travail, ont fait l'objet d'une saisie informatique sur le logiciel Aglaé. Une base administrative d'accords fut ainsi constituée et a permis d'étudier les modalités de RTT, l'équilibre financier des accords ou les engagements en termes d'emploi engagements transformés aussitôt par la ministre en embauches effectives lui permettant d'affirmer, lors de conférences de presse régulières, que la RTT créait des milliers d'emploi.

En cela, l'évaluation hautement politique de la première loi Aubry, puisqu'elle devait légitimer l'efficacité de l'action engagée et appuyer l'adoption de normes précises par la seconde loi, a fait l'objet d'un usage décisionniste et technocratique, bien plus que pragmatique ${ }^{35}$. Celui-ci a même été contes-

34. Antoine JeAmmaud et Évelyne SERVERIN, « Évaluer le droit », Recueil Dalloz Sirey, 1992, Chr. LII 263, p. 266.

35. Reprenant des catégories avancées par Jürgen Habermas, on peut en effet distinguer une évaluation décisionniste (l'expert évalue, mais c'est le politique qui prend les décisions), technocratique (ce sont les experts, s'appuyant sur leur évaluation, qui prennent les décisions et les imposent aux politiques) ou pragmatique (c'est à la suite d'un débat public autour des conditions et résultats de l'évaluation que sont prises les décisions). 
té par le service des statistiques du ministère du Travail lui-même en mai 1999 36. Un bilan a finalement été réalisé et rendu public en septembre 1999, au moment où s'ouvrait le débat parlementaire sur la seconde loi. Et s'il n'a pas été entaché de toute tentative d'instrumentalisation, il s'est avéré précieux : les moyens engagés par l'État pour suivre la mise en œuvre de la mesure ont permis la construction de données et d'analyses nombreuses, dont les publications, bien que retardées après la seconde loi, ont informé en détail sur la réforme en cours et ses premiers effets en termes d'emplois crées, de négociations, de règles adoptées dans les entreprises ${ }^{37}$.

À côté de cette base informatique et de sondages effectués parfois de manière hâtive auprès de chefs d'entreprise ou de salariés, plusieurs enquêtes ont été diligentées pour analyser les processus de RTT. "L'enquête $500 »(1999)$ puis «l'enquête $1000 »(2001)$, par exemple, renseignent sur le point de vue des employeurs, tandis que la seule enquête statistique sérieuse menée auprès des salariés (l'enquête "RTT et Mode de vie ») a eu lieu en 2001. Quant au dispositif Sidonie, il correspond à un registre tenu par l'Agence nationale pour l'amélioration des conditions de travail (ANACT) et était destiné à valider l'activité des consultants, dont une partie des prestations étaient prises en charges par l'État lors des passages aux 35 heures. Il n'a toutefois pas fait l'objet d'exploitation avant le 21 avril 2002 et le changement de cap des politiques publiques qui en résulte en matière de réduction du temps de travail. De son côté, l'analyse statistique des bases d'accords 35h s'appuie sur une nouvelle base. Aglaé étant arrêté dès 2001, un autre registre prend alors le relais : celui construit par l'URSSAF qui collecte les cotisations sociales et par là dispose d'informations sur les entreprises demandant les allègements de charge ouverts par le passage négocié aux 35 heures. On voit ici comment des enjeux annexes (le recouvrement des prélèvements obligatoires) peuvent donner naissance à une base statistique mais aussi comment les variations de l'action publique rendent incertaine la continuité de ces registres susceptibles d'ouvrir une évaluation plus ou moins instrumentale des modes d'appropriation, de diffusion et « d'application » des lois et du droit.

\section{II.3. La problématisation de la mesure des litiges: la tradition des enquêtes sur le contentieux (litigation studies)}

Les questionnements suscités par la problématique de l'évaluation du droit apparaissent ainsi multiples et complexes. Après avoir distingué un certain nombre de grands enjeux du débat scientifique et évoqué des exemples de dispositifs institutionnels de mesure statistique, nous proposons à présent de partir de quelques questions méthodologiques ciblées pour illus-

36. En effet, la DARES a refusé de signer un pré-bilan écrit au forceps en mai 1999 par le cabinet du ministre. Voir la contribution de Catherine Bloch-London à la journée d'étude « Statistiques publique, évaluation et démocratie », 21 mars 2001 (http://cgtinsee.free.fr/Kolok/).

37. Voir notamment les numéros spéciaux de Travail et Emploi, 82 et 83, 2000.
Prendre la mesure du droit : enjeux de l'observation statistique pour la sociologie juridique 
trer de quelle manière la littérature de sociologie du droit a pu mettre en œuvre, dans des protocoles d'enquêtes déterminés, les enjeux généraux de l'observation quantitative de l'activité juridique. C'est assurément le domaine constitué par l'étude du contentieux (notamment le contentieux civil) qui historiquement a donné lieu, dans le champ de la sociologie du droit, à la tradition la plus riche en investigations statistiques, en particulier dans le monde anglo-saxon. Les exemples de questions et controverses méthodologiques que nous évoquerons brièvement relèvent de trois catégories: la plus générale renvoie à l'analyse de la population des affaires traitées par les tribunaux du point de vue de la structure des flux, la seconde est centrée sur la mesure du taux de recours à la justice, la dernière enfin s'interroge sur les profils d'usagers du tribunal.

La question de la répartition des flux d'affaires et de leur évolution dans le temps renvoie à deux types de variables auxquelles s'intéresse la recherche empirique : celles relatives à la procédure et celles qui renvoient aux droits substantiels. Dans une enquête devenue classique, qui les a conduits à dépouiller les archives judiciaires de deux tribunaux californiens, représentatives d'un siècle de procédures, Lawrence M. Friedman et Robert Percival 38 s'appuient sur les variables procédurales pour mettre en évidence un phénomène de «bureaucratisation » ou de «routinisation » de l'activité des tribunaux: les nombreux abandons de procédure, le taux important de décisions favorables au demandeur, le faible degré de motivation des décisions (variables "procédurales» au sens large) en sont autant d'indices. L'analyse par nature d'affaires (variables relatives aux droits substantiels) montre, quant à elle, une tendance historique au recul des contentieux, qui n'est pas sans lien avec la mise en place de techniques d'évitement du tribunal, notamment par les acteurs économiques (dispositifs de prévention de l'impayé ou de règlement non juridictionnel des litiges liés au recouvrement de créances).

De leur côté, les enquêtes par analyse longitudinale des flux d'affaires ont amené les recherches empiriques sur le contentieux à problématiser la question plus spécifique du taux de recours à l'institution judiciaire, indicateur dont les difficultés de construction et d'interprétation donnent lieu à controverses et soulignent d'autant plus la complexité des débats autour des notions de « juridicisation » ou de « judiciarisation » 39 . Les résultats de la recherche de Friedman et Percival montrent de ce point de vue que l'estimation du recours au tribunal a davantage de force explicative lorsqu'elle s'effectue par nature d'affaires plutôt que de manière globale. En effet, l'évolution des volumes d'affaires apparaît différenciée suivant la nature des droits substantiels en jeu: l'augmentation des affaires liées au

38. Lawrence Friedman et Robert Percival, «A Tale of Two Courts : Litigation in Alameda and San Benito Counties », Law and Society Review, 10 (2), 1976, p. 267-301.

39. Pour une discussion récente de ces notions, cf. Jacques CommaILlE et Martine KALUSZYNSKI (dir.), La fonction politique de la justice, Paris, La Découverte, coll. « Recherche », 2007. 
droit de la famille, pour lesquelles la puissance publique rend incontournable un enregistrement et une homologation administrative par l'institution judiciaire, est régulière et importante ; à l'inverse, la décrue est nette pour ce qui est des affaires ressortant du contentieux contractuel.

Une fois posé le principe d'une étude par situation d'action juridique, se pose le problème plus aigu de la définition d'une population de référence pour l'estimation d'un taux de recours au juge. En réalité, la signification de l'effectif de référence choisi varie extrêmement suivant que l'on définit cette population comme correspondant (1) au nombre de relations juridiques ; (2) au nombre de personnes engagées dans une relation juridique ; (3) au nombre de litiges nés de ces relations ; (4) au nombre de règlements, quelle que soit leur nature (contractuelle ou judiciaire), qui sont engagés pour traiter ces litiges. Parmi ces définitions, seules les deux premières semblent pouvoir relever d'un enregistrement institutionnel (données de type « registres »), les deux autres ne pouvant a priori être construites que par enquêtes auprès des usagers du droit. Ces différentes définitions ne sont pas a priori exclusives les unes des autres, mais peuvent suivant les situations d'action correspondre à des logiques distinctes. Ainsi, deux estimations rapportant sur une période donnée le nombre de demandes adressées aux Conseils de prud'hommes en matière de licenciement, dans le premier cas, au stock des contrats de travail actuellement en cours (population active du secteur privé) et, dans le second cas, au nombre de ruptures de contrat de travail notifiées sur cette période, correspondent simplement à des mesures de type différent : la première donnera l'image d'une " population soumise au risque », tandis que la seconde sera davantage centrée sur la mesure du taux de recours stricto sensu.

En outre, l'exemple du contentieux du travail est relativement simple, dans la mesure où la plupart des salariés n'ont qu'un employeur. Si l'on s'intéresse au contentieux des contrats en général (baux, ventes et prêts, prestations de services ou contrats commerciaux), la situation est plus complexe dans la mesure où une même personne, physique ou morale, peut être engagée dans des obligations de nature diverse, concernant des formes contractuelles qui ne font pas systématiquement l'objet, comme les statistiques du travail, de modes d'enregistrement institutionnalisés. Les protocoles d'enquête doivent alors se mettre en quête d'indicateurs indirects ou partiels : une étude statistique réalisée en Espagne s'appuyant sur des données relatives aux effectifs d'actes notariés a ainsi pu mettre en évidence un seuil de croissance similaire à celui montré par Friedman et Percival concernant le taux de recours au juge en matière contractuelle. Sur une longue période, la croissance des actes notariés (considérée comme indicateur possible de recours au droit) croît plus vite que la croissance du conten-
Prendre la mesure du droit : enjeux de l'observation statistique pour la sociologie juridique 
tieux contractuel. C'est ainsi un phénomène de juridicisation sans judiciarisation que l'on pourrait constater 40 .

Outre la difficulté de construction de ces indicateurs, les controverses portent également sur leur interprétation délicate qui renvoie plus fondamentalement à la conception des rapports entre droit et société. Comme le montre l'étude mentionnée précédemment, on peut raisonnablement supposer que le déclin de certains domaines d'affaires, jadis gros pourvoyeurs de procès, n'est pas lié à un reflux dans l'absolu du nombre de litiges qui, dans les sociétés modernes, opposent entre elles au quotidien des personnes, morales et physiques. Un moindre recours au procès ne signifie donc pas un moindre recours au droit, mais un changement dans les usages du droit.

Pour Friedman et Percival, le fait que les affaires traitées par les juges soient de plus en plus routinières, d'une part, et qu'un seuil semble avoir été atteint dans la croissance des recours aux tribunaux, d'autre part, participe d'une réduction du rôle des tribunaux dans le règlement des litiges. À l'inverse, Richard Lempert discute dans une controverse célèbre l'interprétation des résultats de cette enquête en réfutant son hypothèse implicite qui pose comme équivalente la diminution sur le plan quantitatif du contentieux des contrats et la perte d'influence de la fonction judiciaire de règlement des litiges. L'impact des décisions judiciaires sur les pratiques contractuelles peut en effet a contrario fournir un élément d'explication quant à la diminution du contentieux. La stabilisation des règles par la mise au point d'une jurisprudence et la menace d'une sanction crédible et prévisible sont autant d'effets s'exerçant à la fois sur la production des outils juridiques en amont et sur les règlements des litiges 41 .

La dernière catégorie d'exemples relatifs aux méthodologies d'enquêtes mises en œuvre renvoie à l'étude des profils d'usagers du droit, en l'occurrence les pourvoyeurs de procès. Sur le plan théorique, ce type d'enquêtes se rattache aux idéaux-types proposés par Marc Galanter dans son étude sur les caractéristiques des usagers du droit, dans laquelle il oppose en particulier les usagers occasionnels du droit (one-shotters) aux usagers récurrents (repeat players). Ces derniers disposent en effet d'avantages comparatifs liés à une dotation supérieure en ressources économiques, institutionnelles et cognitives et à un engagement routinier et plus ou moins professionnalisé dans le maniement des instruments juridiques (l'archétype de l'usager récurrent étant l'agent d'assurance ou le directeur de personnel) ${ }^{42}$. De nombreuses études statistiques se sont inspirées de ce cadre théorique, mettant ainsi en évidence l'existence de clientèles régulières des tribunaux

40. José TOHARIA, «Social Legal Activity in Spain 1900-1970», Yale Program in Law and Modernisation, 24, 1973.

41. Richard LEMPERT, « More Tales of Two Courts : Exploring Changes in the "Dispute Settlement Function" of Trials Courts », Law and Society Review, 13 (1), 1978, p. 91-138.

42. Marc GALANTER, "Why the "Haves" Come Out Ahead : Speculations on the Limits of Legal Change », Law and Society Review, 9 (1), 1974, p. 95-160. 
attachées à des profils spécifiques d'affaires, eux-mêmes corrélés à des profils de décisions judiciaires. Les affaires de recouvrement de l'impayé par les sociétés de crédit, dont l'issue est le plus souvent favorable au demandeur face à des défendeurs peu combatifs ou absents, en fournissent notamment l'image la plus connue ${ }^{43}$.

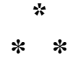

Au-delà de ces exemples, qui positionnent le plus souvent l'analyse quantitative comme un élément particulier, unique ou le plus souvent partiel, de preuve d'un raisonnement sociologique sur le droit, rappeler en quelques mots les principaux éléments de la boîte à outils comprenant des données et des méthodes statistiques peut être utile pour prendre la mesure des données quantitatives construites autour ou à partir d'" objets juridiques ». En forme de conclusion, on peut ainsi rappeler que l'analyse de données quantitatives est particulièrement ajustée pour mettre en évidence des régularités, informer sur des évolutions ou souligner la stabilité de déterminants sociaux - tous phénomènes qui intéressent au plus haut point le sociologue soucieux de déconstruire les pré-notions et de construire un point de vue général. Cependant, la méthode statistique est un outil d'exploration et pas seulement de preuve à propos des processus sociaux que les sociologues du droit peuvent chercher à comprendre et expliquer. Il serait en effet dommageable de n'inscrire ces données quantitatives que dans des raisonnements " toutes choses égales par ailleurs », où sont recherchés des déterminants causaux, et dont l'identification des influences propres ne préjuge ni de leur combinaison ni des manières dont les acteurs construisent la réalité sociale par leurs représentations, leur définition des situations et leurs pratiques plus ou moins quotidiennes ou exceptionnelles.

L'opposition soulignée par Alain Desrosières entre logique monographique (qui comprend l'analyse factorielle représentant des espaces sociaux peuplés d'individus en relations) et logiques explicatives (qui transforment ces individus en variables et cherchent à en expliquer les déterminants par des régressions) 44 constitue ainsi probablement deux manières de construire la totalité qu'il faut maintenir pour étudier ces activités juridiques et judiciaires, qu'elles soient institutionnalisées ou non, qu'elles laissent ou non des traces susceptibles de quantification, qu'elles fassent l'objet d'enregistrements routinisés ou nécessitent des enquêtes spécifiques. Si mise en

43. Craig WANNER, «The Public Ordering of Private Relations. Part Two : Winning Civil Court Cases », Law and Society Review, 9 (2), 1975, p. 293-306 ; Stanton WHEELER, Bliss CARTWRIGHT, Robert KAGAN et Lawrence FrIEDMAN, « Do the "Haves" Come Out Ahead? Winning and Losing in State Supreme Courts, 1870-1970 », Law and Society Review, 21 (3), 1987, p. 403-446.

44. Alain DESROSIÈRES, «L'opposition entre deux formes d'enquête : monographie et statistique », in Luc BOLTANSKI et Laurent THEVENOT (dir.), Justesse et justice dans le travail, Paris, PUF, coll. « Cahiers du Centre d'études de l'emploi », 1989.
Prendre la mesure du droit : enjeux de l'observation statistique pour la sociologie juridique 
forme légale et mise en forme statistique ont de telles proximités et si le recours aux données quantitatives apparaît si crucial pour certains raisonnements, sans qu'elles soient pour autant toujours mobilisées, c'est bien que ce registre d'exploration et de démonstration mérite toute sa place, dans la sociologie en général comme dans la sociologie des activités juridiques et judiciaires. 